\title{
New tools for diet analysis: nanopore sequencing of metagenomic DNA from rat stomach contents to quantify diet
}

\author{
Nikki Freed ${ }^{1}$, William Pearman ${ }^{1}$, Adam Smith ${ }^{1}$, Georgia Breckell ${ }^{1}$, James Dale ${ }^{1}$, and Olin \\ Silander ${ }^{1}$
}

${ }^{1}$ Massey University - Albany Campus

September 9, 2020

\begin{abstract}
Accurate determination of animal diets is difficult. Methods such as molecular barcoding or metagenomics offer a promising approach, allowing quantitative and sensitive detection of different taxa. Here we show that rapid and inexpensive diet quantification is possible through metagenomic sequencing with the portable Oxford Nanopore Technologies (ONT) MinION. Using an amplification-free approach, we profiled the stomach contents from 24 wild-caught rats. We conservatively identified diet items from over 50 taxonomic orders, ranging across nine phyla, including plants, vertebrates, invertebrates, and fungi. This highlights the wide range of taxa that can be identified using this simple approach. We calibrated the accuracy of this method by comparing the characteristics of reads matching the ground-truth host genome (rat) to those matching diet items, and show that at the family-level, taxon assignments are approximately $97.5 \%$ accurate. Some inaccuracies may arise from database biases; we suggest a way to mitigate for database biases when using metagenomic approaches. Finally, we implemented a constrained ordination analysis and show that we can identify the sampling location of an individual rat within tens of kilometres based on diet content alone. This work establishes proof-of-principle for long-read metagenomic methods in quantitative diet analysis. We show that diet content can be quantified even with limited expertise, using a simple, amplification free workflow and a relatively inexpensive and accessible next generation sequencing method. Continued increases in the accuracy and throughput of ONT sequencing, along with improved genomic databases, suggests that a metagenomic approach for quantification of animal diets will become an important method in the future.
\end{abstract}

\section{Hosted file}

Rat Paper_Ecol_Evol_2020-09-09.pdf available at https://authorea.com/users/357514/articles/ 480026-new-tools-for-diet-analysis-nanopore-sequencing-of-metagenomic-dna-from-ratstomach-contents-to-quantify-diet 